\title{
An Atmospheric Pressure Ion Lens that Improves Nebulizer Assisted Electrospray Ion Sources
}

\author{
Bradley B. Schneider, D. J. Douglas, and David D. Y. Chen \\ Department of Chemistry, University of British Columbia, Vancouver, British Columbia, Canada
}

An atmospheric pressure ion lens improves the performance and ease of use of a nebulizer assisted electrospray (ion spray) ion source. The lens is comprised of an oblong-shaped stainless steel ring attached to an external high voltage power supply. The lens is located near the tip of the conductive sprayer, and is maintained at a potential less than that of the sprayer. The ion lens improves the shape of the equipotential lines in the vicinity of the sprayer tip. This lens gives approximately a 2-fold reduction in the signal RSD, a 2-fold increase in the ion signal, an increase in the number of multiply charged ions, and a much broader range of usable sprayer positions. (J Am Soc Mass Spectrom 2002, 13, 906-913) (c) 2002 American Society for Mass Spectrometry

$\mathrm{E}$ lectrospray ionization (ESI), first demonstrated by Fenn and co-workers [1], is a versatile technique for the formation of gas phase ions for mass spectrometry. In these early studies, the sprayer was stainless steel syringe tubing with an internal diameter of $100 \mu \mathrm{m}$, and the solution flow rates were a few $\mu \mathrm{L} / \mathrm{min}$ or greater. Since then, ESI has been used extensively for many applications including analysis of drugs [2], proteins [3], and oligonucleotides [4], to name a few. A key concern in many of these types of analyses is maximizing the magnitude and stability of the ion signal.

Electrospray ion sources can be operated over a wide range of solution flow rates, including high flow rate $(\geq 1 \mu \mathrm{L} / \mathrm{min})$, reduced flow rate $(80 \mathrm{~nL} / \mathrm{min}-1 \mu \mathrm{L} /$ $\mathrm{min})$, and very low flow rate $(<80 \mathrm{~nL} / \mathrm{min})$. Wilm and Mann described the first ESI source for operation at very low liquid flow rates $(\approx 20 \mathrm{~nL} / \mathrm{min})$ [5]. Stabilization of electrospray at this flow rate required the use of tapered capillary tips, with internal diameters of 1-2 $\mu \mathrm{m}$. A thin layer of gold was deposited on the glass capillary tips for application of the electrospray potential. This technique was termed nanoelectrospray ionization. Sampling efficiency was increased, however operation with the tapered tips was more difficult than electrospray at higher flow rates.

The first description of electrospray operating as a reduced flow rate ion source was by Smith and coworkers [6], in which a capillary electrophoresis system was coupled with a mass spectrometer. Shortly thereafter,

Published online June 14, 2002

Address reprint requests to Dr. D. D. Y. Chen, 2036 Main Mall, Department of Chemistry, University of British Columbia, Vancouver, BC, V6T 1Z1, Canada. E-mail: chen@chem.ubc.ca
Emmett et al. described a packed electrospray needle for interfacing chromatography with mass spectrometry at low flow rates (300 $\mathrm{nL} / \mathrm{min}$ and above) [7]. Following these initial experiments, there have been many studies with electrospray operating in this flow regime for applications involving direct infusion [8,9], or the coupling of CE $[10,11]$ and LC $[12,13]$ with ESI-MS. Typically these reduced flow rate ESI sources provide improved signal stability and sampling efficiency when compared to higher flow rate ESI sources. However they require the use of tapered capillaries with a metal coating [5], incapillary electrode [14], or some other type of junction [8, $15,16]$ for applying the electrospray potential.

High flow rate electrospray ion sources are easy to operate, but typically suffer from a lower sampling efficiency and poorer signal stability when compared with reduced flow rate electrospray ion sources. This problem is accentuated when spraying samples with high aqueous content. A nebulizer can be used to help break up droplets formed at the sprayer tip [17]. This technique of nebulizer assisted electrospray ionization, or "ion spray" helps to stabilize the ESI process for flow rates up to approximately $1 \mathrm{~mL} / \mathrm{min}$.

Recently we described a device for stabilizing and increasing the ion signals from reduced flow rate electrospray ion sources [18]. The signal improvement was obtained by placing an ion lens around the tapered tip of the electrospray capillary, leading to an improvement in the shape of the equipotentials near the sprayer tip. Note this method improves sensitivity by focusing ions at atmospheric pressure rather than in one of the differentially pumped vacuum stages $[19,20]$. A reviewer has brought it to our attention that Micromass has used a lens to enhance the performance of ion 
transmission in their Z-SPRAY API source (Micromass, Manchester, UK). This is a deflection lens located downstream from the electrospray capillary, rather than a focusing lens located behind the tip of the electrospray capillary. The work presented in this paper is an extension of the ion lens technology to the $1-5 \mu \mathrm{L} / \mathrm{min}$ flow rate regime. The lens is found to improve the stability of the ion signals substantially, while also increasing the magnitude of the signal of an ion spray source with flow rates of $1-5 \mu \mathrm{L} / \mathrm{min}$. In addition, the ion spray source is much easier to operate when the lens is in place because the changes in the ion signal with different sprayer positions are greatly reduced. The lens is also found to increase the ratio of multiply charged to singly charged ions. The performance enhancements generated by the ion lens should be applicable to even higher flow rates, such as those compatible with HPLC-MS.

\section{Experimental}

The acetate salt of bradykinin of $99 \%$ purity and $\beta$-cyclodextrin of $98 \%$ purity were from Sigma (St. Louis, $\mathrm{MO})$. Various concentrations of $\beta$-cyclodextrin were prepared by dissolution of the sugar in water with 0.01 $M$ certified ACS grade ammonium acetate from BDH Chemicals (Toronto, ON, Canada). HPLC grade methanol and glacial acetic acid were from Fisher Scientific Ltd. (Nepean, ON, Canada). Bradykinin was prepared by dissolution of the peptide salt in a solution of $59.5 \%$ water, $39.5 \%$ methanol, and $1 \%$ acetic acid at concentrations ranging from $10^{-4}$ to $10^{-6} \mathrm{M}$.

The instrument used in this study was a prototype triple quadrupole mass spectrometer from SCIEX (Concord, ON, Canada). The ion sampling interface and ion optics are similar to those of the SCIEX API 300 system. The ion spray needle and mount shown in Figure 1a were built in-house. Sample was delivered to the sprayer through a fused silica capillary (Polymicro Technologies, Phoenix, AZ) with a $150 \mu$ m o.d. and a 50 $\mu \mathrm{m}$ i.d. The fused silica capillary was inserted into two concentric stainless steel tubes (Small Parts Inc., Miami Lakes, FL) with standard wall thickness for 19 and 27 gauge, respectively (referred to as ion spray configuration 1). For some experiments, the 19 gauge tube was replaced with a smaller diameter 21 gauge stainless steel tube (referred to as ion spray configuration 2). A stainless steel tee (Valco Instruments, Houston, TX) was used to hold the sprayer in place, and allow attachment of the nebulizer gas flow line. The nebulizer gas was compressed air (Praxair, Mississauga, ON, Canada, medical grade). The electrospray potential was applied through the mounting bracket to the stainless steel tee. The ion lens was composed of an oblong-shaped aperture cut into a rectangular piece of stainless steel with 1 $\mathrm{mm}$ thickness. An arm on the mounting bracket was used to adjust the position of the ion lens (Figure $1 \mathrm{~b}$ ) relative to the sprayer. For these experiments, the lens was set back $2 \mathrm{~mm}$ from the tip of the sprayer. The lens was oriented perpendicular to the axis of the stainless
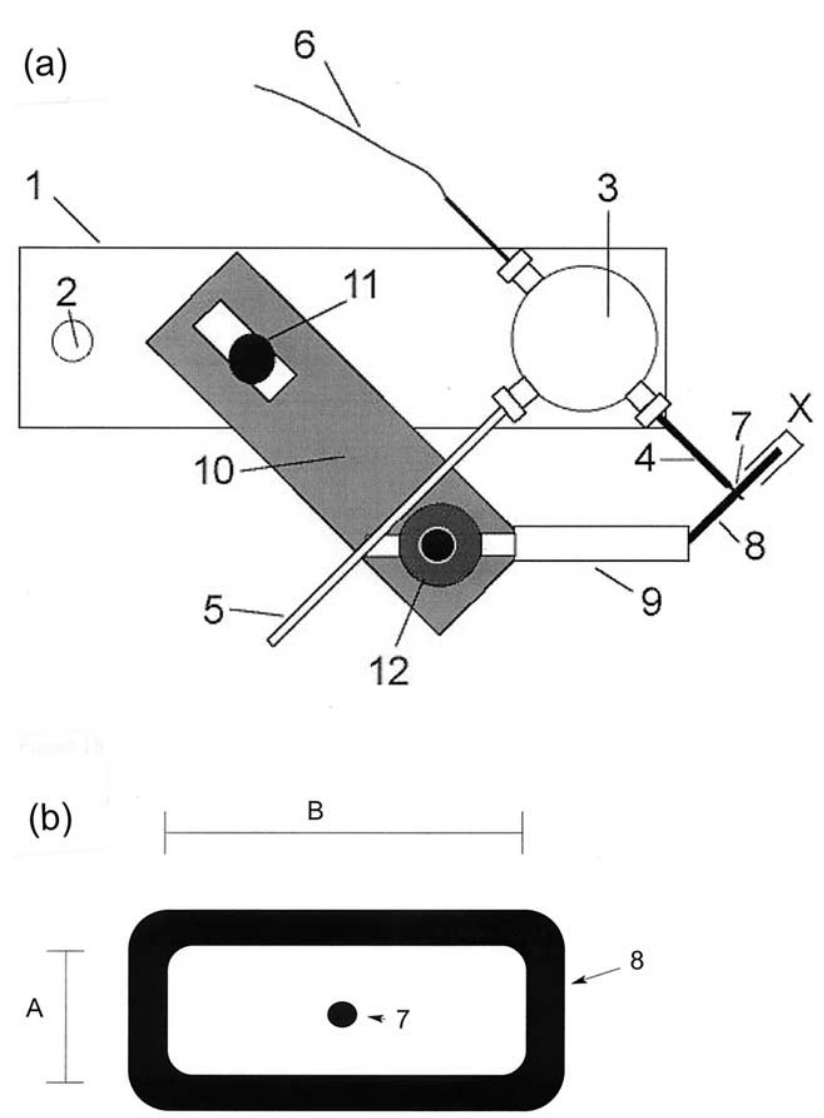

Figure 1. (a) Schematic of the ion spray source. The ion spray mount (1) is attached to the mass spectrometer with a stud inserted through the mounting hole (2). The electrospray potential is applied to a stainless steel tee (3) through the conductive mount (1). Two concentric stainless steel capillaries (4) are used for the sample and the nebulizer gas flowing through the nebulizer gas line (5). The sample is introduced through a fused silica capillary (6) to the inner stainless steel tube (7). The ion lens (8) is located near the tip of the inner stainless steel tube (7). The ion lens has a mounting bracket (9) and an adjustable arm (10). Pivots (11 and 12) allow the lens to be positioned in a range of different locations. The length of protrusion of the inner stainless steel tube from the outer tube is labeled " $\mathrm{X}$ ". (b) Front view of the ion lens (8) demonstrating the location of the stainless steel sprayer tube (7) within the lens. The vertical (A) and horizontal (B) dimensions of the aperture in the lens (8) are shown.

steel sprayer as shown in Figure 1a. Different lens sizes were constructed by varying the aperture diameter in the vertical dimension (A) and the horizontal dimension (B). A Spellman CZE 1000R (Hauppauge, NY) power supply provided the voltage to the lens. Ultrahigh-purity nitrogen from Praxair (Mississauga, ON, Canada, manufacturer's stated purity $99.9995 \%$ ) was used as the curtain gas for the triple quadrupole system, and the solution flow rate was controlled by a syringe infusion pump (Model 22, Harvard Apparatus, South Natick, MA).

\section{Results and Discussion}

The mechanism of formation of ions from charged droplets in ESI is unresolved. Two mechanisms have 

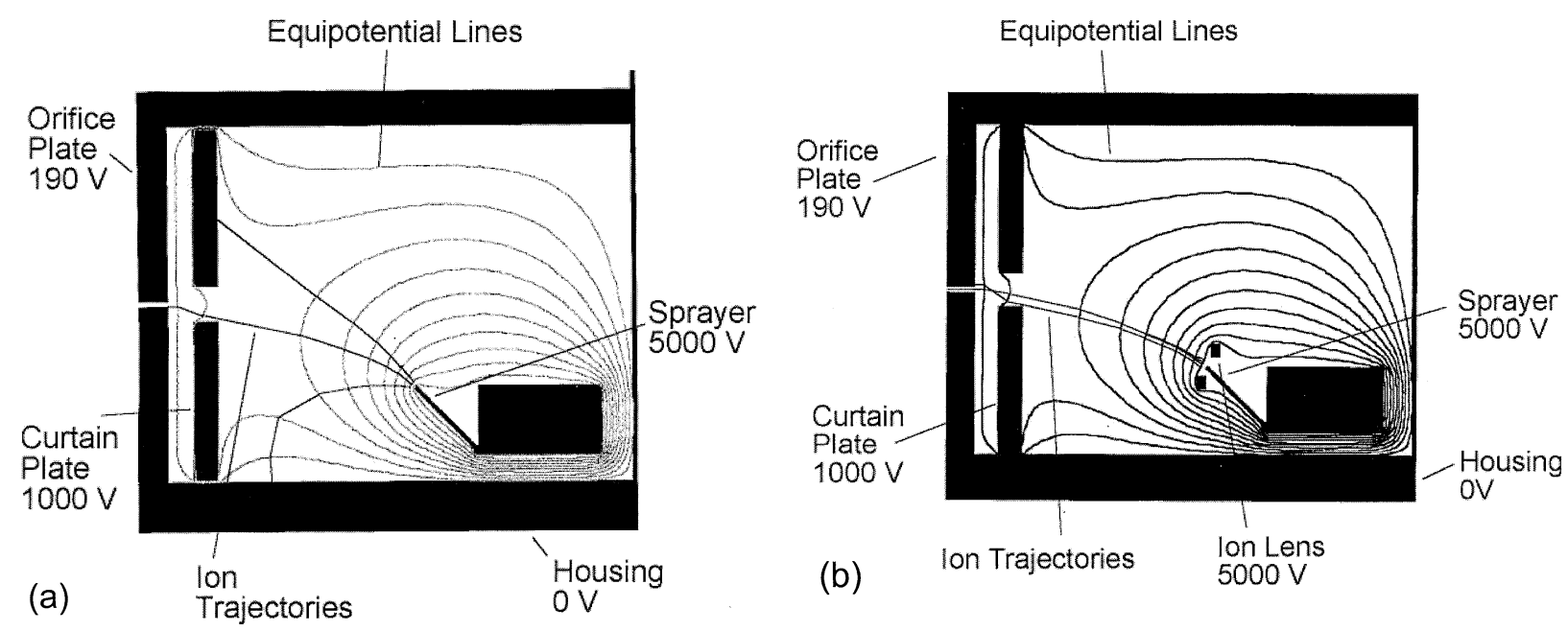

Figure 2. Schematics of the equipotential lines generated for a standard ion spray source (a) and an ion spray source with the ion lens (b). Potentials are listed in the figure. The contour interval is approximately $500 \mathrm{~V}$.

been proposed. The first, proposed by Iribarne and Thomson, involves ion evaporation from small microdroplets with radii less than $1 \mu \mathrm{m}$ [21]. The microdroplets may be formed by Coulumbic fissions of larger droplets with an excess of charge above the Rayleigh limit of instability [21]. The second mechanism, proposed by Rollgen and co-workers, involves the release of single ions from very small droplets (diameter $\approx 1$ $\mathrm{nm}$ ) by solvent evaporation [22]. Other research groups have also attempted to describe the mechanisms involved in the formation of ions by electrospray [23-26]. Although the proposed mechanisms have slight differences, the one similarity is that a mixture of ions and charged droplets is generated in the atmospheric pressure region between the ESI sprayer and the entrance aperture to the mass spectrometer.

In the absence of any gas flow, ions and small charged droplets at atmospheric pressure have trajectories orthogonal to the electric equipotential lines. The ion drift velocity $(\vec{v})$ is given by:

$$
\vec{\nu}=k \vec{E}
$$

where $\vec{E}$ is the electric field and $k$ is the mobility [27]. The use of a nebulizer to aid in break-up of the charged droplets produced by an ion spray source generates an additional velocity vector to complicate the description of the motion of small droplets. Figure 2a shows the equipotential lines for a typical nebulizer assisted electrospray ion source. The curtain plate, orifice plate, and housing serve as counter electrodes for the ESI source. The region between the curtain plate and the orifice plate is at atmospheric pressure and is flushed with nitrogen gas. The rest of the interior of the housing is also at atmospheric pressure. The orifice plate separates the atmospheric pressure region in the housing from the first stage of the vacuum system of the mass spectrometer. The potentials in this figure are $5000 \mathrm{~V}$,
$1000 \mathrm{~V}, 190 \mathrm{~V}$, and $0 \mathrm{~V}$ for the sprayer, curtain plate, orifice plate, and housing, respectively. Qualitative ion trajectories (drawn by hand) illustrate that a wide plume of ions is generated, resulting in a low efficiency of transfer of ions to the mass spectrometer. Figure $2 \mathrm{~b}$ demonstrates the improvement in the shape of the equipotential lines near the tip of the ion spray source when an atmospheric pressure ion lens is incorporated. The potentials in this figure are $5000 \mathrm{~V}, 5000 \mathrm{~V}, 1000 \mathrm{~V}$, $190 \mathrm{~V}$, and $0 \mathrm{~V}$, for the sprayer, ion lens, curtain plate, orifice plate, and housing, respectively. The equipotential lines near the tip of the ion spray source are flattened, reducing the ion defocusing effect. Qualitative ion trajectories (drawn by hand) demonstrate that a greater number of ions is directed toward the inlet aperture of the mass spectrometer.

Prior to testing of the ion lens, the configuration of the ion spray tip was optimized for the two different sprayers by adjustment of the length with which the innermost stainless steel tube protrudes from the outer tube (" $\mathrm{X}$ " in Figure 1a). This parameter was found to be most important for signal stability and magnitude with ion spray configuration 2 (21 gauge middle tube). The length, $X$, was varied from 1 to $5 \mathrm{~mm}$, and the sprayer, lens, and curtain plate voltages, as well as the nebulizer gas flow were optimized to produce the maximum ion signal for the doubly charged peak of the peptide bradykinin with both ion spray configurations. The results are shown in Figure 3. Ion spray configuration 1 was found to yield a stronger and more stable ion signal than configuration 2 . The signal for configuration 1 was independent of $X$ over the range 1-5 $\mathrm{mm}$ with a nebulizer pressure of 5 psi. For configuration 2, the maximum ion signal was obtained with $\mathrm{X}$ adjusted to the range between 2 and $3 \mathrm{~mm}$ with a nebulizer pressure of 45 psi. Shorter or longer values resulted in attenuation of the ion signal by a factor of approximately 2 . For the data presented in this paper, $\mathrm{X}$ values 


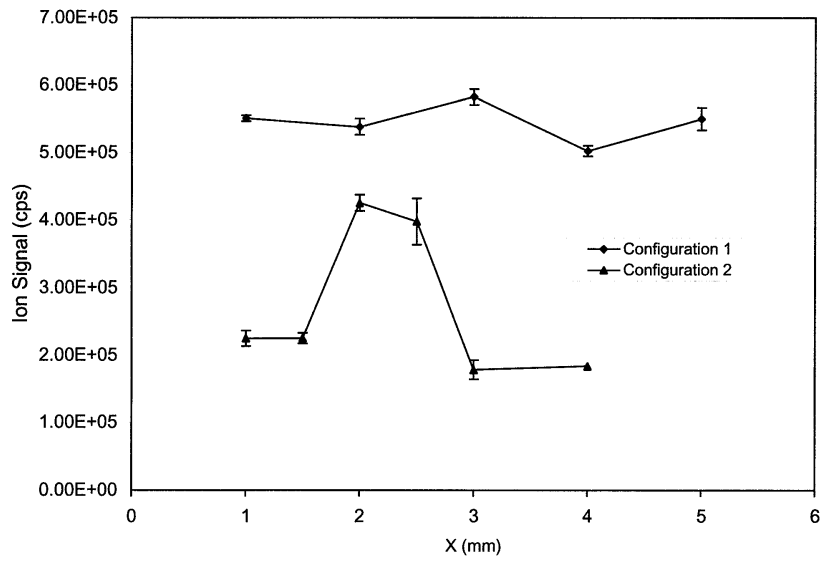

Figure 3. The signal for doubly protonated bradykinin versus the length of the inner stainless steel tube protruding from the outer tubing.

of 3 and $2 \mathrm{~mm}$ were used to optimize the signal magnitude for configurations 1 and 2, respectively.

Various lens dimensions were used in these studies. The best results were achieved with oblong-shaped ion lenses. Lens dimensions utilized in these studies are listed in Table 1. It is important to note that lenses with dimensions smaller than lens 1 were susceptible to arcing between the lens and the sprayer. The lenses were positioned perpendicular to the sprayer. For incorporation of lenses 1 through 3 into the ion source, the sprayer was oriented at approximately a $45^{\circ}$ angle to the curtain plate. For lenses 4 and 5 , the sprayer had to be turned on a $90^{\circ}$ angle (perpendicular to the face of the curtain plate) to prevent arcing between the edge of the ion lens and the curtain plate during optimization of the sprayer position. Similar signal magnitudes and stabilities were obtained with all of the oblong-shaped lenses.

The optimum position for the lens with both ion spray configurations was approximately $2 \mathrm{~mm}$ behind the sprayer tip. As the lens was moved closer to the sprayer tip, there was an attenuation of the ion signal, most likely due to a decrease in the magnitude of the electric field near the sprayer tip. This is similar to what was previously observed with a reduced flow rate electrospray ion source, except that in this case the nebulizer gas blows the solution away from the sprayer tip regardless of the magnitude of the electric field [18]. The effects of the lens were also attenuated as it was moved farther back from the sprayer tip, even when the

Table 1. Dimensions of the oblong-shaped ion lenses used in these studies

\begin{tabular}{lrr}
\hline Lens & $\mathrm{A}(\mathrm{mm})$ & $\mathrm{B}(\mathrm{mm})$ \\
\hline \hline 1 & 8.90 & 12.40 \\
2 & 10.20 & 14.10 \\
3 & 11.10 & 14.92 \\
4 & 13.00 & 17.60 \\
5 & 15.00 & 19.30 \\
\hline
\end{tabular}

lens potentials were reoptimized. For the results presented in this paper, the lens was positioned $2 \mathrm{~mm}$ behind the sprayer tip with both ion spray configurations.

The ion lens provides a substantial improvement in the stability of the ion signal for an ion spray source. Data collected for repeat measurements of the magnitude of the singly charged peak for a sample of $10^{-4} \mathrm{M}$ $\beta$-cyclodextrin were compared. The potential applied to the detector was adjusted to obtain similar signal magnitudes with and without the ion lens. The RSD of either 6 or 20 individual measurements was calculated. Each measurement of the signal was determined by the addition of 10 scans with a $10 \mathrm{~ms}$ dwell time, and the peak height was used. The signal RSD was approximately 1.4 and $3.0 \%$ for the ion spray source with and without the lens, respectively. For comparison, the RSD expected from count statistics was $0.5 \%$. The signal RSD was reduced by a factor of approximately 2 with the addition of the ion lens. The signal stability improvement with the ion lens can be important for analyses such as determination of isotopic peak ratios for ions.

The ion lens increases the magnitude of the ion signal detected within a mass spectrometer. Typical signal increases range from a minimum of approximately 1.5 times up to a maximum of approximately 4 times. To achieve these results, it was found that elevated curtain plate potentials were important. Typical potentials applied to the curtain plate were $1600-$ $2000 \mathrm{~V}$. There was no indication of arcing in the front end of the mass spectrometer with these elevated curtain plate potentials.

The sensitivity gains with the ion lens are accentuated for higher charge states. Figure 4 shows the results for analysis of a $10^{-6} \mathrm{M}$ sample of bradykinin. The mass spectrum in Figure 4a shows the signal obtained for doubly $(\mathrm{m} / \mathrm{z}=531)$ and triply protonated $(\mathrm{m} / \mathrm{z}=354)$ peptide with $6627 \mathrm{~V}, 1000 \mathrm{~V}, 1790 \mathrm{~V}$, and $140 \mathrm{~V}$ applied to the sprayer, ion lens, curtain plate, and orifice, respectively. The mass spectrum in Figure $4 \mathrm{~b}$ was obtained with the same sample, and the same potentials except that $3500 \mathrm{~V}$ was applied to the ion lens. The increase in lens potential resulted in an increase in the magnitude of all of the doubly and triply charged peptide peaks observed in the mass spectrum, however, the background noise was unaffected. In some cases, the background signal from singly charged ions decreased with higher lens potentials. This charge state effect can be useful for analysis of low concentrations of analytes that produce multiply charged ions, such as peptides.

The variation of signal for doubly and triply protonated bradykinin with increasing lens potential is presented in Figure 5 for a $10^{-4} \mathrm{M}$ sample of the peptide. The maximum ion signal obtained with ion spray was approximately $1.00 \times 10^{6} \mathrm{cps}$ for the doubly protonated ion and $1.82 \times 10^{5} \mathrm{cps}$ for the triply charged ion. The addition of the ion lens increased the number of doubly and triply protonated ions detected in the mass spec- 

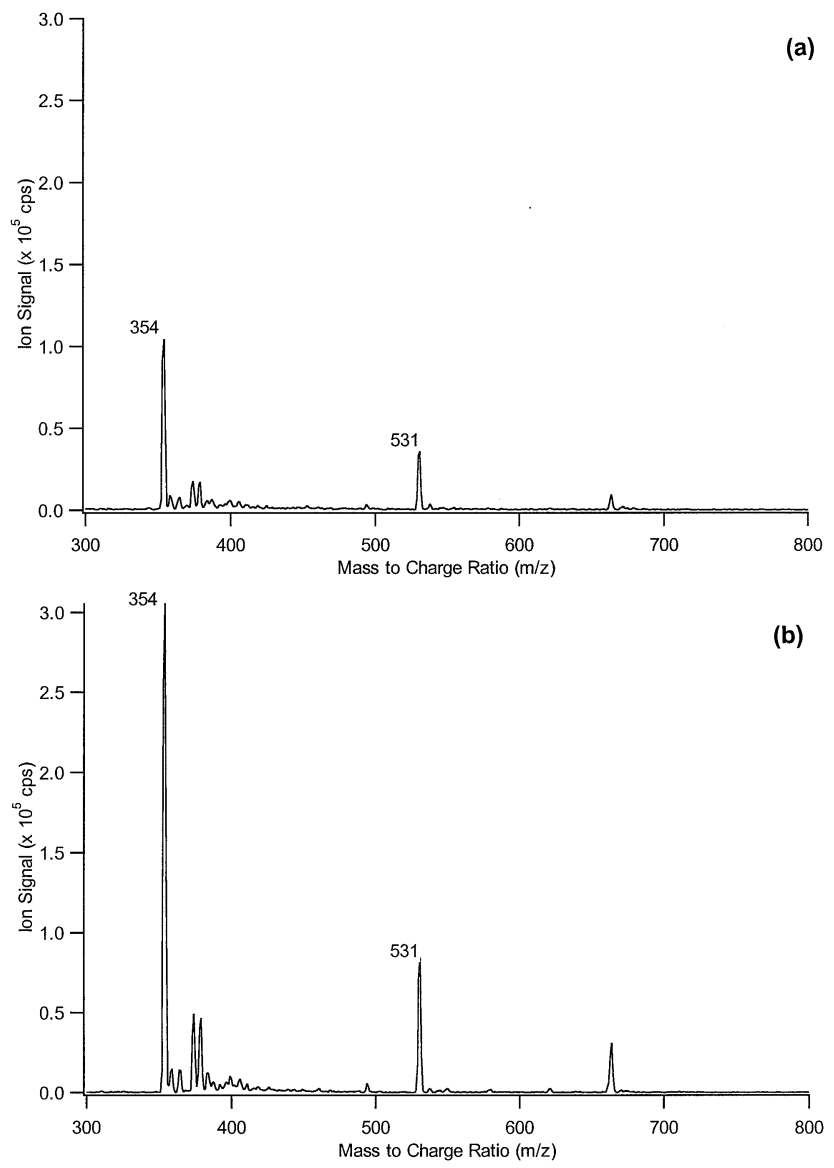

Figure 4. Mass spectra of bradykinin demonstrating the increase in the magnitude of the multiply charged peaks as the ion lens potential is increased from $1000 \mathrm{~V}$ (a) to $3500 \mathrm{~V}(\mathbf{b})$. The magnitude of the multiply charged analyte peaks is increased, but the background is not.

trometer. The increase to the doubly charged ion is only a factor of 1.4, but the increase to the triply charged ion is a factor of approximately 7 . As the lens potential is increased, the doubly and triply charged ion signals have maxima at different potentials. Ions of higher

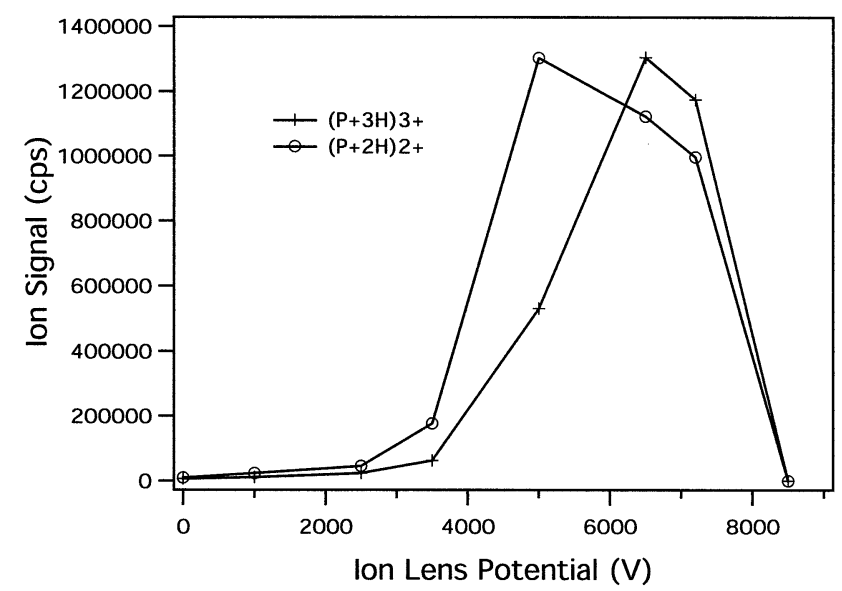

Figure 5. The ion signal for doubly (o) and triply $(+)$ protonated bradykinin versus the ion lens potential.

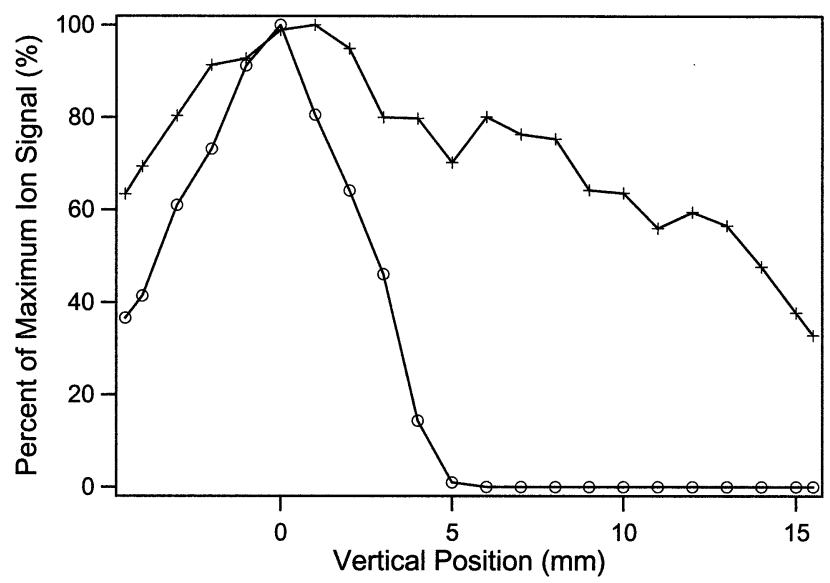

Figure 6. The magnitude of the ion signal for doubly protonated bradykinin as the sprayer is moved away from the optimum location for ion spray with $(+)$ and without $(o)$ the ion lens. The sprayer is moved in the vertical direction from the optimum location at a height even with the aperture in the curtain plate.

charge states are optimized with an increased lens potential. It is interesting to note that the significant increase in the magnitude of the signal for the triply charged ion is observed with only a minor decrease in the magnitude of the signal for the doubly charged ion. This shows that more ions are focused into the entrance aperture of the mass spectrometer, most likely due to the improved shape of the equipotential lines. It is also possible the potential from the lens somehow changes the spray process so that more highly charged ions are formed. At very high lens potentials (above or close to the potential applied to the sprayer) the signal from all ions can be completely eliminated.

The ion signal exhibits a reduced dependence on sprayer position with the lens in place. The dependence of the signal magnitude on the vertical position of the sprayer is demonstrated in Figure 6 for ion spray with and without the atmospheric pressure ion lens. Successful operation of this ion spray source requires careful positioning of the probe in the vertical direction. For the data of this figure, the sprayer was optimized approximately $1.5 \mathrm{~cm}$ from the curtain plate, and $1 \mathrm{~mm}$ off to the right hand side of the aperture in the curtain plate with a $10^{-4} \mathrm{M}$ sample of bradykinin. The voltages for the sprayer, ion lens, and curtain plate were $6000 \mathrm{~V}$, $2400 \mathrm{~V}$, and $1970 \mathrm{~V}$, respectively. For the runs without the ion lens, the voltages for the sprayer and curtain plate were $6000 \mathrm{~V}$ and $1100 \mathrm{~V}$, respectively. A vertical position of $0 \mathrm{~mm}$ is defined as the point where the sprayer tip is the same height as the middle of the aperture in the curtain plate. The signal with and without the lens is normalized to 100 at this point. Positions above the optimum location are defined as positive, and positions below the optimum location are defined as negative. The maximum range of adjustment available with this housing was $-5.5 \mathrm{~mm}$ to $+15.5 \mathrm{~mm}$. Movement of the sprayer $3 \mathrm{~mm}$ up or down from the optimum position attenuated the ion signal by a factor 


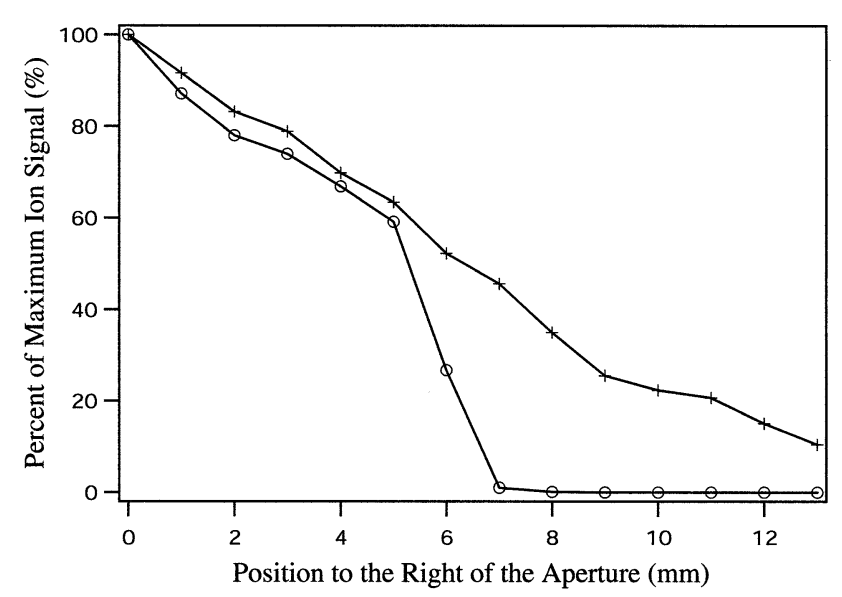

Figure 7. The magnitude of the ion signal for doubly protonated bradykinin as the sprayer is moved away from the optimum location for ion spray with (+) and without (o) the ion lens. The sprayer is moved to the right hand side of the aperture in the curtain plate, and the source potentials are not reoptimized at each position.

of slightly greater than 2 in the absence of the ion lens. Elevation of the sprayer by $6 \mathrm{~mm}$ completely eliminated the signal for doubly protonated bradykinin using the ion spray source. The installation of the ion lens leads to a dramatic decrease in the dependence of the ion signal on vertical position. The ion signal is attenuated by a factor of 2 only after the probe is raised $14 \mathrm{~mm}$ from the optimum position. Greater than $35 \%$ of the initial ion signal is maintained at a sprayer position $15.5 \mathrm{~mm}$ above the optimal location. If a lens is present on a mass spectrometer, an ion signal can be observed regardless of whether the sprayer position is optimized or not.

Moving the sprayer a few millimeters in the horizontal direction from the optimum location can also make a substantial difference in the magnitude of the observed ion signal with this ion spray source. The use of the ion lens near the tip of the sprayer diminishes this effect. A broader range of sprayer positions is available for maintaining strong ion signals. Figure 7 demonstrates this phenomenon for movement of the sprayer off to the right hand side of the aperture in the curtain plate with a sample of $10^{-4} \mathrm{M} \beta$-cyclodextrin in water with $5 \mathrm{mM}$ ammonium acetate. The voltage parameters were the same as those utilized for the data in Figure 6. The point defined as $0 \mathrm{~mm}$ is the edge of the curtain plate to the right of the aperture. The signals with and without the lens are normalized to 100 at this point. The sprayer was moved in $1 \mathrm{~mm}$ increments to the right of the aperture in the curtain plate. The sprayer was positioned approximately $1.5 \mathrm{~cm}$ from the curtain plate. Initially, with the sprayer in the optimum location, there is approximately 1.5 times more signal obtained for the singly protonated cyclodextrin molecule with the ion lens in place. When the sprayer is moved off to the right hand side, the signal decreases both with and without the lens. The signal has completely disappeared for the ion spray source when it is moved $8 \mathrm{~mm}$

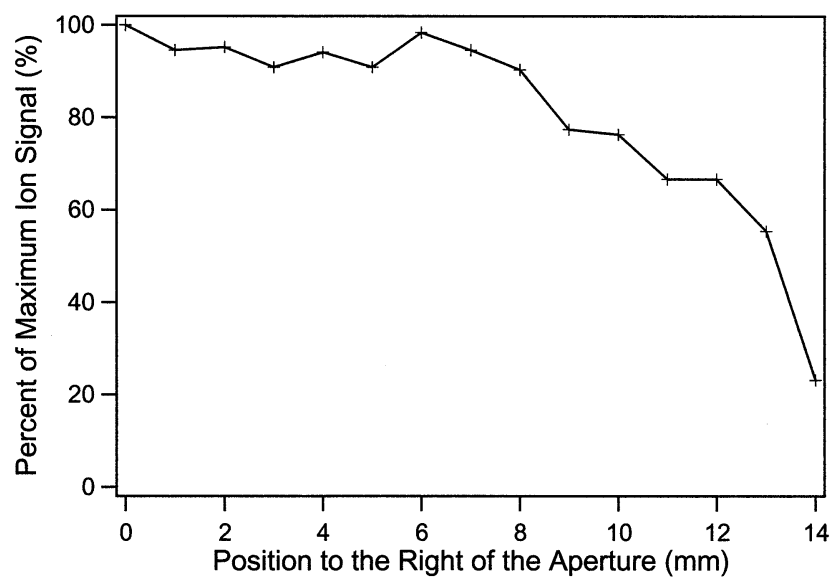

Figure 8. The ion signal as the ion spray source with the ion lens is moved away from the optimum location. The sprayer is moved to the right hand side of the aperture in the curtain plate, and the source potentials are reoptimized at each position.

from the optimum location. For the sprayer with the ion lens, signal is maintained for distances up to $14 \mathrm{~mm}$ from the optimum location. It is important to note that the signal with the lens in place is greater than the maximum ion spray signal up to approximately $4 \mathrm{~mm}$ from its optimum location. As the sprayer is positioned closer to the curtain plate, the effect becomes even more dramatic. The ion signals increase as the sprayer is positioned closer to the entrance aperture of the instrument. However, with the ion spray source, the loss of signal is much more dramatic as the sprayer is moved to the right hand side. The decreased dependence of ion signal on the sprayer position has important implications in the areas of high throughput analysis and separations integrated with mass spectrometry. Sprayer position can be difficult to optimize in situations where capillary electrophoresis or liquid chromatography are interfaced to ESI-MS. Currently, success in these types of analyses is dependent upon the proper location of the sprayer. The lens provides an inexpensive performance enhancement for any electrospray source, and may be particularly useful in reducing the sprayer positional requirements for analyses involving separation techniques interfaced to ESI-MS.

The data in Figures 6 and 7 demonstrate that the ion lens provides a much wider range of useful positions for generating strong ion signals with an ion spray source, however, this data was gathered without trying to reoptimize the source lens potentials at each of the positions. Figure 8 demonstrates the signal obtained for the ion spray source with an ion lens as the sprayer is moved off to the right hand side of the aperture in the curtain plate for doubly protonated ions from a $10^{-4} \mathrm{M}$ sample of bradykinin. In this experiment, the source potentials were reoptimized at each position. Greater than $90 \%$ of the initial ion signal was maintained as the sprayer was moved $8 \mathrm{~mm}$ from the optimum location, compared to slightly less than $50 \%$ of the original ion signal in Figure 7 without reoptimization of the source 


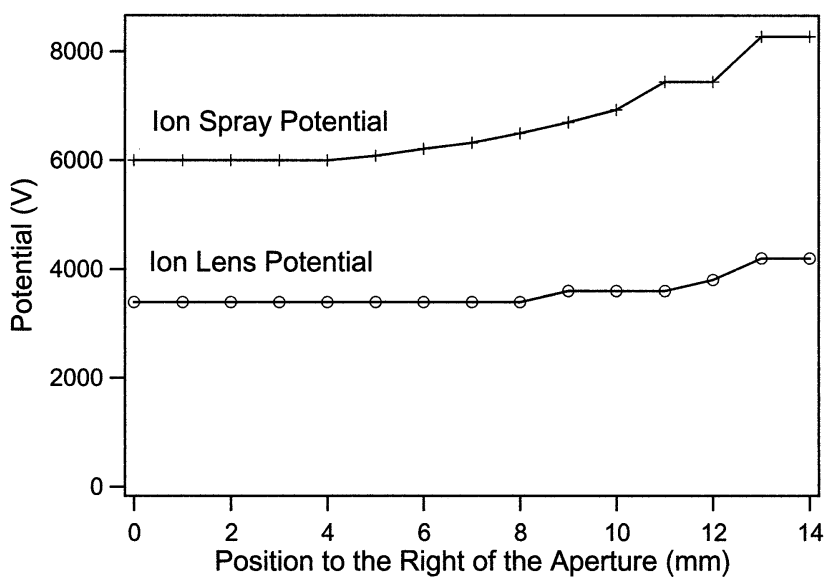

Figure 9. The optimized potentials applied to the sprayer and the ion lens for the data presented in Figure 8.

potentials. When the source potentials were reoptimized at each position, greater than $50 \%$ of the initial ion signal was maintained even though the sprayer was moved $13 \mathrm{~mm}$ from the optimum position. Optimization of the source potentials involved increasing the sprayer and ion lens potential as the sprayer was moved farther from its initial location. The curtain plate potential was approximately constant. Figure 9 shows the optimized sprayer and ion lens potential for each of the sprayer positions with respect to the aperture in the curtain plate. It is important to note that at sprayer potentials above $8200 \mathrm{~V}$, arcing was observed in the source region. This limitation led to the dramatic decrease in the ion signal as shown in Figure 8 at a position $14 \mathrm{~mm}$ to the right of the aperture in the curtain plate.

\section{Conclusion}

It is shown that the incorporation of an ion lens located near the tip of a nebulizer assisted electrospray ion source improves its performance substantially. The lens increases and stabilizes the ion signals, particularly for ions of higher charge states. In addition, the lens makes an ion spray source easier to use by reducing the positional dependence of the ion signal. A strong ion signal can still be observed with the sprayer positioned at surprisingly large distances $(>1 \mathrm{~cm})$ from the optimum location. The reduced sprayer positional dependence may be useful for stabilization of multiple sprayer electrospray ion sources. In addition, the ability to turn off a sprayer with high lens potential (Figure 5) may permit electronic control of multiple sprayers.

\section{Acknowledgments}

This work was supported by the Natural Sciences and Engineering Research Council of Canada (NSERC) and Applied Biosystems/MDS SCIEX. BBS acknowledges a post-graduate scholarship from NSERC. DJD acknowledges an Industrial Research Chair supported by NSERC and SCIEX.

\section{References}

1. Yamashita, M.; Fenn, J. B. Another Variation on the Free-Jet Theme. J. Phys Chem. 1984, 88, 4451-4459.

2. Weinmann, W.; Wiedemann, A.; Eppinger, B.; Renz, M.; Svoboda, M. Screening for Drugs in Serum by Electrospray Ionization/Collision-Induced Dissociation and Library Searching. J. Am. Soc. Mass Spectrom. 1999, 10, 1028-1037.

3. Chowdury, S. K.; Katta, V.; Chait, B. T. Probing Conformational-Changes in Proteins by Mass-Spectrometry. J. Am. Chem. Soc. 1990, 112, 9012-9013.

4. Deforce, D. L.; Van den Eeckhout, E. C. Analysis of Oligonucleotides by ESI-MS. Adv. Chromatogr. 2000, 40, 539-566.

5. Wilm, M.; Mann, M. Analytical Properties of the Nanoelectrospray Ion Source. Anal. Chem. 1996, 68, 1-8.

6. Wahl, J. H.; Goodlett, D. R.; Udseth, H. R.; Smith, R. D. Attomole Level Capillary Electrophoresis Mass-Spectrometric Protein-Analysis Using $5 \mu \mathrm{m}$-I.D. Capillaries. Anal. Chem. 1992, 64, 3194-3196.

7. Emmett, M. R.; Caprioli, R. M. Micro-Electrospray Mass-Spectrometry-Ultra-High-Sensitivity Analysis of Peptides and Proteins. J. Am. Soc. Mass Spectrom. 1994, 5, 605-613.

8. Figeys, D.; Aebersold, R. High Sensitivity Identification of Proteins by Electrospray Ionization Tandem Mass Spectrometry: Initial Comparison Between an Ion Trap Mass Spectrometer and a Triple Quadrupole Mass Spectrometer. Electrophoresis 1997, 18, 360-368.

9. Schneider, B. B.; Chen, D. D. Y. Collision-Induced Dissociation of Ions Within the Orifice-Skimmer Region of an Electrospray Mass Spectrometer. Anal. Chem. 2000, 72, 791-799.

10. Mazereeuw, M.; Hofte, A. J. P.; Tjaden, U. R.; van der Greef, J. A Novel Sheathless and Electrodeless Microelectrospray Interface for the On-Line Coupling of Capillary Zone Electrophoresis to Mass Spectrometry. Rapid Commun. Mass Spectrom. 1997, 11, 981-986.

11. Bateman, K. P.; White, R. L.; Thibault, P. Evaluation of Adsorption Preconcentration Capillary Zone Electrophoresis Nanoelectrospray Mass Spectrometry for Peptide and Glycoprotein Analyses. J. Mass Spectrom. 1998, 33, 1109-1123.

12. Alexander, J. N., IV; Schultz, G. A.; Poli, J. B. Development of a Nano-Electrospray Mass Spectrometry Source for Nanoscale Liquid Chromatography and Sheathless Capillary Electrophoresis. Rapid Commun. Mass Spectrom. 1998, 12, 1187-1191.

13. Le Bihan, T.; Pinto, D.; Figeys, D. Nanoflow Gradient Generator Coupled with $\mu$-LC-ESI-MS/MS for Protein Identification. Anal. Chem. 2001, 73, 1307-1315.

14. Smith, A. D.; Moini, M. Control of Electrochemical Reactions at the Capillary Electrophoresis Outlet/Electrospray Emitter Electrode under CE/ESI-MS through the Application of Redox Buffers. Anal. Chem. 2001, 73, 240-246.

15. Severs, J. C.; Smith, R. D. Characterization of the Microdialysis Junction Interface for Capillary Electrophoresis Microelectrospray Ionization Mass Spectrometry. Anal. Chem. 1997, 69, 2154-2158.

16. Zhang, B.; Foret, F.; Karger, B. L. A Microdevice with Integrated Liquid Junction for Facile Peptide and Protein Analysis by Capillary Electrophoresis/Electrospray Mass Spectrometry. Anal. Chem. 2000, 72, 1015-1022.

17. Bruins, A. P.; Covey, T. R.; Henion, J. D. Ion Spray Interface for Combined Liquid Chromatography Atmospheric Pressure Ionization Mass Spectrometry. Anal. Chem. 1987, 59, 26422646.

18. Schneider, B. B.; Douglas, D. J.; Chen, D. D. Y. An Atmospheric Pressure Ion Lens to Improve Electrospray Ionization at Low Solution Flow-Rates. Rapid Commun. Mass Spectrom. 2001, 15, $2168-2175$ 
19. Mylchreest, I. C.; Hail, M. E.; Herron, H. R. Method and Apparatus for Focusing Ions in the Viscous Flow Jet Expansion Region of an Electrospray Apparatus. U.S. Patent Number 5,157,260, filed May 17, 1991.

20. Belov, M. E.; Gorshkov, M. V.; Udseth, H. R.; Anderson, G. A.; Tolmachev, A. V.; Prior, D. C.; Harkewicz, R.; Smith, R. D. Initial Implementation of an Electrodynamic Ion Funnel with Fourier Transform Ion Cyclotron Resonance Mass Spectrometry. J. Am. Soc. Mass Spectrom. 2000, 11, 19-23.

21. Iribarne, J. V.; Thomson, B. A. On the Evaporation of Small Ions from Charged Droplets. J. Chem. Phys. 1976, 64, 22872294.

22. Schmelzeisen-Redeker, G.; Butfering, L.; Rollgen, F. W. Desolvation of Ions and Molecules in Thermospray Mass Spectrometry. Int. J. Mass Spectrom. Ion Processes 1989, 90, $139-150$.
23. Ikonomou, M. G.; Blades, A. T.; Kebarle, P. Electrospray Ion Spray-A Comparison of Mechanisms and Performance. Anal. Chem. 1991, 63, 1989-1998.

24. Kebarle, P.; Tang, L. From Ions in Solution to Ions in the Gas-Phase-The Mechanism of Electrospray Mass-Spectrometry. Anal. Chem. 1993, 65, 972A-986A.

25. Siu, K. W. M.; Guevremont, R.; Le Blanc, J. C. Y.; O'Brien, R. T.; Berman, S. S. Is Droplet Evaporation Crucial in the Mechanism of Electrospray Mass-Spectrometry? Org. Mass Spectrom. 1993, $28,579-584$.

26. Hager, D. B.; Dovichi, N. J.; Klassen, J.; Kebarle, P. Droplet Electrospray Mass-Spectrometry. Anal. Chem. 1994, 66, 39443949.

27. Asbury, G. R.; Hill, H. H., Jr. Using Different Drift Gases to Change Separation Factors $(\alpha)$ in Ion Mobility Spectrometry. Anal. Chem. 2000, 72, 580-584. 